\title{
Low-Grade Urothelial Carcinoma: Reappraisal of the Cytologic Criteria on ThinPrep ${ }^{\circledR}$
}

\author{
Wei Xin, M.D., Ph.D. ${ }^{1}$ Stephen S. Raab, M.D. ${ }^{2}$ and Claire W. Michael, M.D. ${ }^{1 *}$
}

The diagnostic criteria for low-grade urothelial lesions that have been described in the past were based on urinary specimens prepared by the cytospin method. Recognizing the recent popularity of the ThinPrep ${ }^{\circledast}$ methodology and the cytologic alterations it introduces to the cellular features, we sought to evaluate the reproducibility of these criteria in ThinPrep urinary samples. One hundred twenty-six ThinPrep urinary specimens with a tissue diagnosis of low-grade urothelial carcinoma (LGUC) and 45 negative controls were evaluated. Three pathologists blindly reviewed the slides separately and the consensus on each feature was used in the study. Logistic regression analysis was used to determine which criteria in combination were most predictive of low-grade urothelial carcinoma. All specimens were evaluated for the following 18 features: nucleus/cytoplasm ratio, irregular nuclear border, cytoplasm homogeneity, cell clusters, high cellularity, prominent nucleoli, granular nuclear chromatin, hyperchromasia, acute inflammation, vesicular chromatin, nuclear molding, nuclear eccentricity, elongated nuclei, necrosis, anisonucleosis, irregular bordered fragments, absent cytoplasmic collar, and peripheral palisading. High nucleus-to-cytoplasm ratio, irregular nuclear borders, and homogeneous cytoplasm (combination sensitivity of 59\% and specificity of 100\%) were the best predictive features for LGUC. Minor predictive criteria were eccentric nuclei and nuclear molding. ThinPrep provides well preserved, cleaner specimens without significantly altering the morphology. The three key criteria applied in cytospin specimens to diagnose LGUC were reproducible in ThinPrep specimens. Diagn. Cytopathol. 2003; 29:125-129. ๑ 2003 Wiley-Liss, Inc.

Key Words: low-grade urothelial carcinoma; ThinPrep; urine; cytology

Bladder cancers account for 12,600 deaths and more than 56,500 new cases per year, with a male-to-female ratio of 2.7:1. ${ }^{1}$ More than $90 \%$ of the bladder cancers are urothelial carcinomas. The papillary carcinomas outnumber the flat

\footnotetext{
${ }^{1}$ Department of Pathology, University of Michigan Medical Center, Ann Arbor, Michigan

${ }^{2}$ University of Pittsburgh, Pittsburgh, Pennsylvania

*Correspondence to: Claire W. Michael, M.D., Department of Pathology, University of Michigan Hospitals, 1500 E. Medical Center Dr., Ann Arbor, MI 48109. E-mail: clairemi@med.umich.edu

Received 27 December 2002; Accepted 3 April 2003

DOI 10.1002/dc. 10311

Published online in Wiley InterScience (www.interscience.wiley.com).
}

ones. The papillary urothelial tumors usually present as low grade at early stage and involve multiple sites. Although they often recur, they can be easily managed by local excision. ${ }^{2,3}$ According to the 1998 World Health Organization and International Society of Urological Pathologists classification, papillary urothelial neoplasms are classified as papilloma, urothelial carcinoma with low malignant potential, low-grade urothelial carcinoma, and high-grade urothelial carcinoma. ${ }^{4}$

Diagnosing low-grade urothelial carcinoma (LGUC) on cytologic specimen is very difficult, because the tumor cells are well differentiated, they may exhibit only a slight degree of atypia, and they closely resemble normal urothelial cells. Separating them from reactive, regenerative and reparative changes is not always possible. Increased nuclear-to-cytoplasmic (N/C) ratio, irregular nuclear membrane, and cytoplasmic homogeneity were three key features reported by Raab et al. ${ }^{5,6}$ for diagnosing LGUC in cytospin specimens.

ThinPrep ${ }^{\circledR}$ preparation (Cytyc, Boxborough, MA) was initially tested on nongynecologic specimens, as a monolayer preparation, and was then introduced as an alternative to the conventional preparation for cervical smear to remove obscuring elements and improve the diagnosis. ${ }^{7,8}$ In recent years, many laboratories have implemented the ThinPrep method in preparing nongynecologic specimens. The ThinPrep method provides a clear background with the absence of blood, cell debris, and inflammatory cells. However, ThinPrep alters the cellular morphology when compared with conventional preparations. Some of the reported cytologic alterations of ThinPrep specimens that may affect the interpretation of LGUC include fragmented papillary clusters, more single cells with apparent discohesion, cells appearing smaller and spindly, enhanced nucleoli, increased cytoplasmic density, and less vesicular chromatin.9,10 In the present study, we undertook to evaluate those previously described features in ThinPrep specimens and the most important cytologic features for diagnosing LGUC. 
Table I. Original Cytologic Diagnosis in Biopsy-confirmed Low-Grade Urothelial Carcinomas and Benign Changes

\begin{tabular}{lcclccr}
\hline & \multicolumn{2}{c}{ LGUC $^{a}$} & & \multicolumn{2}{c}{ Benigh $^{b}$} & \\
\cline { 2 - 3 } & Positive $^{c}$ & Negative $^{d}$ & & Positive $^{c}$ & Negative $^{d}$ & Total \\
\hline Washing $^{\mathrm{e}}$ & 23 & 27 & & 0 & 17 & 67 \\
Voided & 14 & 62 & & 0 & 28 & 104 \\
\hline
\end{tabular}

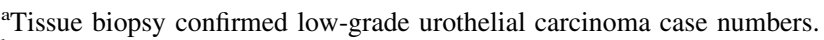

${ }^{\mathrm{b}}$ Tissue biopsy confirmed benign lesion case numbers.

'Positive cases includes both positive and suspicious for urothelial carcinoma.

${ }^{\mathrm{d}}$ Negative cases include atypical cells present and negative for carcinoma.

${ }^{\mathrm{e}}$ All washing specimens including catheter-collected specimens.

\section{Materials and Methods}

\section{Cases}

The cytology pathology files at the University of Michigan Medical Center were reviewed retrospectively for the years 1995-2001. The selected ThinPrep cases included 49 bladder washings and 76 void urine specimens with corresponding tissue diagnosis of LGUC within 6 mo. Patient ages ranged from 41 to $88 \mathrm{yr}$ of age, with a mean of $56 \mathrm{yr}$. In addition, 17 bladder washings and 28 voided urine samples with corresponding tissue diagnosis of inflammation, reactive, or regenerative features within 6 mo were selected as the control group. Patient ages ranged in age from 40 to 85 $\mathrm{yr}$, with a mean of $54 \mathrm{yr}$. Table I summarizes the original cytology diagnoses.

\section{Evaluation}

Three pathologists blindly reviewed each case separately and evaluated the selected previously described eighteen cytologic features. These features are increased N/C ratio, irregular nuclear border, cytoplasm homogeneity, cell clusters, high cellularity, prominent nucleoli, granular nuclear chromatin, hyperchromasia, acute inflammation, vesicular chromatin, nuclear molding, nuclear eccentricity, elongated nuclei, necrosis, anisonucleosis, fragments with irregular border, absent cytoplasmic collar (cytoplasmic rim between the nucleus and cytoplasmic membrane), and peripheral palisading. ${ }^{5,6}$ Samples of the above features are illustrated in Figure C-1. The consensus on each feature in each case was consequently reached. For statistical evaluation, we used the $\chi^{2}$ test or Fisher's exact test (if any observed frequency was $<5)$ to obtain $P$-values. ${ }^{11,12}$

\section{Results}

In biopsy-confirmed LGUC cases, the original diagnosis in ThinPrep washings, based on the three key criteria (increased N/C ratio, irregular nuclear membrane, and cytoplasmic homogeneity), had a sensitivity of $46 \%$ and specificity of $100 \%$. The diagnosis of the ThinPrep voided urine samples gave a sensitivity of $18.4 \%$ and specificity of $100 \%$ (Table II). The washings had a much better sensitivity than voided specimens, when these criteria were used.
Twelve features were statistically significant $(P<$ 0.01 ) in frequency between LGUC and benign changes in both washings and voided urine samples. These twelve features are increased N/C ratio, cytoplasmic homogeneity, irregular nuclear borders, nuclear eccentricity, nuclear molding, anisonucleosis, hyperchromatic nuclear chromatin, elongated nucleus, absent cytoplasmic collar, granular nuclear chromatin, cell clusters, and necrosis (Table III). Overall, the rate of incidence of these twelve features (except for necrosis) was much higher in washings than voided samples. Increased N/C ratio, irregular nuclear border, cytoplasmic homogeneity, and presence of several cell clusters had their highest incidence in the LGUC. Necrosis was present only in carcinoma cases and was rather infrequent.

Table IV summarizes the sensitivity, specificity, positive predictive value and negative predictive value of the statistically significant twelve features for the diagnosis of LGUC. The increased N/C ratio, cytoplasmic homogeneity, and irregular nuclear border had high sensitivities $(67.5 \%$, $65.1 \%$, and $57.1 \%$, respectively) and specificities $(82.2 \%$, $84.4 \%$, and $91.1 \%$, respectively) on ThinPrep specimens. The positive predictive values (PPVs), which were all higher than $90 \%$, were much higher than the negative predictive values, which were all lower than 50\%. Nuclear eccentricity, nuclear molding, and anisonucleosis were also good predictors, with sensitivity and specificity $61.9 \%$ and $82.2 \%, 41.3 \%$ and $93.3 \%$, and $34.1 \%$ and $97.8 \%$, respectively. High cellularity, open chromatin, acute inflammation, irregularly bordered fragments, prominent nucleoli, and peripheral palisading were not statistically significant $(P>0.01)$ either in washings or voided urine samples (Table V).

\section{Discussion}

In urine cytology, high-grade urothelial carcinoma and carcinoma in situ usually do not represent a diagnostic challenge. The urine samples in such cases are characterized by abundant cellularity, isolated and loose clusters of tumor cells with pleomorphism and polymorphism, high N/C ratio, enlarged and eccentric nucleus, hyperchromatic and coarse chromatin, irregular nuclear membrane, and vacuolated cytoplasm. ${ }^{13}$ However, in LGUC, many of the above features do not occur frequently. Histologically, LGUC are characterized by an overall orderly appearance, but with easily recognizable variation of architectural and/or cytologic features. Cytologic atypia usually is definite but minimal, including changes in nuclear size, shape, and chromatin pattern. Therefore, the differentiation between low-grade tumor and benign reactive or reparative change is very difficult on cytologic specimens. ${ }^{4,5,14}$ Increased N/C, irregular nuclear membrane, and cytoplasmic homogeneity have been proposed for diagnosing LGUC and, in combination, 

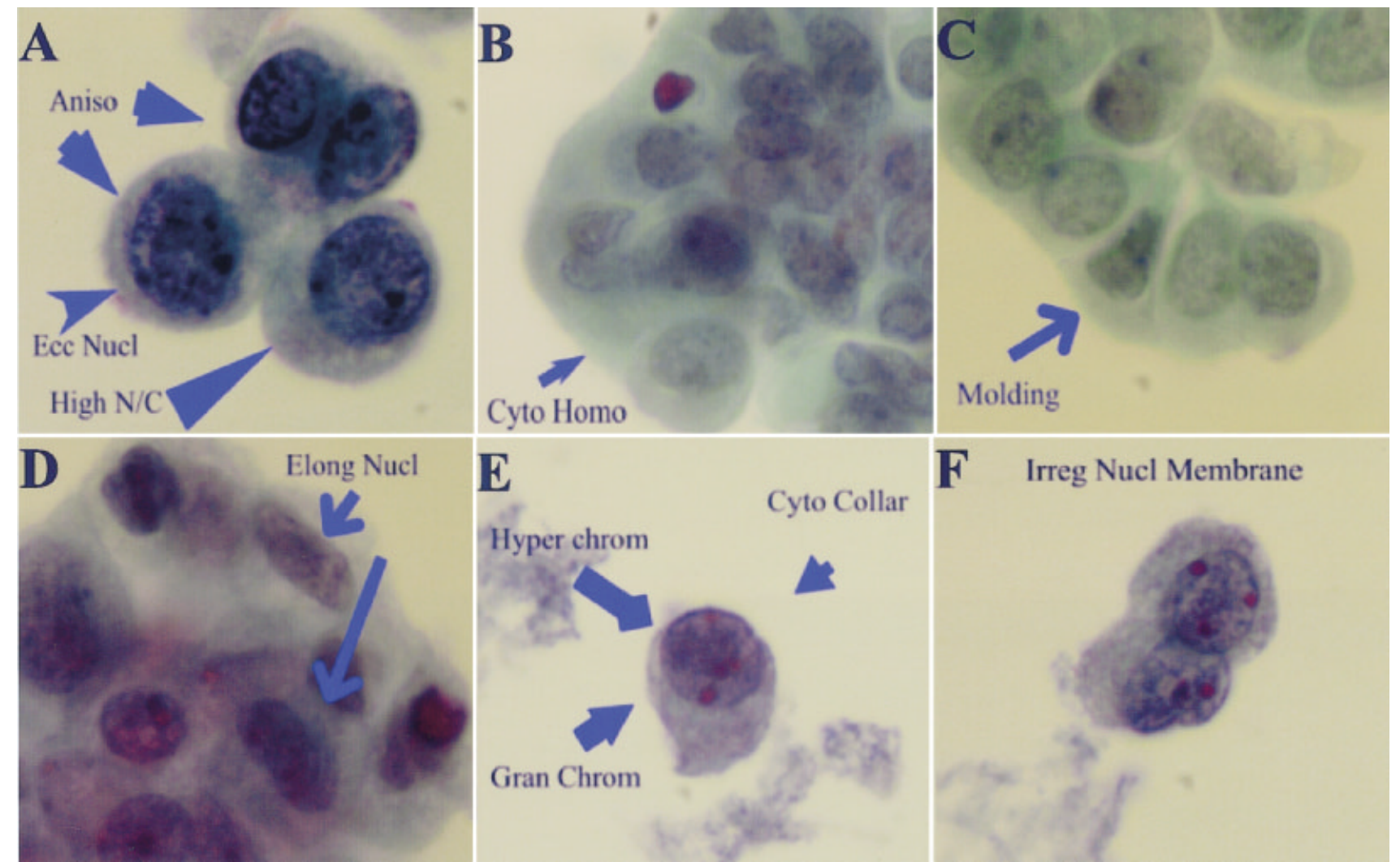

Fig. 1. Selected cytologic features in the diagnosing low-grade urothelial carcinoma in ThinPrep specimens. A: Aniso, anisonucleosis; Ecc Nucl, eccentric nucleus; High N/C, high N/C ratio. B: Cyto homo, cytoplasmic homogeneity. C: Molding, nuclear molding. D: Elong Nucl, elongated nucleus. E: Hyper chrom, hyperchromasia; Cyto collar, absence of cytoplasmic collar; Gran chrom, granular chromatin. F, Irreg Nucl Membrane, irregular nuclear membrane (Papanicolaou stain, $\times 1,000$ ).

Table II. Sensitivity, Specificity, Positive Predictive Value and Negative Predictive Value

\begin{tabular}{lcccc}
\hline & $\begin{array}{c}\text { Sensitivity } \\
(\%)\end{array}$ & $\begin{array}{c}\text { Specificity } \\
(\%)\end{array}$ & $\begin{array}{c}P P V \\
(\%)\end{array}$ & $\begin{array}{c}N P V \\
(\%)\end{array}$ \\
\hline Washing & 46.0 & 100 & 100 & 38.6 \\
Voided & 18.4 & 100 & 100 & 31.1 \\
Combined & 29.4 & 100 & 100 & 33.8 \\
\hline
\end{tabular}

PPV, positive predictive value; NPV, negative predictive value.

these three criteria had a $45 \%$ sensitivity and $98 \%$ specificity in conventional (cytospin) bladder washings. ${ }^{5}$

Urine cytology is still one of the main approaches to follow-up evaluation of patients with urothelial carcinomas. ${ }^{15,16}$ There are two major types of urinary cytologic specimen: bladder washings (including catheterization specimens) and voided urine specimens. Voided urines are easy to obtain, and they represent the entire urinary tract. However, they usually have a low cellularity and carry a high incidence of degeneration and contamination. In contrast, bladder washings have a higher sensitivity, being more cellular with better cell preservation and less contamination. However, their collection is inconvenient and uncomfortable to the patient, and also carries an increased risk of infection. ${ }^{15,17}$ A previous study claimed that ThinPrep could achieve better cellular preservation, cleaner background and high cellularity in voided urine samples compared to conventional preparations. ${ }^{18}$

In this study, we found that increased N/C ratio, irregular nuclear membrane, and cytoplasmic homogeneity were still three key criteria in diagnosing LGUC in ThinPrep samples. In combination, these three features had a sensitivity of 59\% and a specificity of $100 \%$. We noted a slight difference between our sensitivity in the second review and the original cytologic diagnosis, which is not statistically significant (59\% vs 46\%). This is probably due to the variation of different observers' criteria on identifying these cytologic features.

Because urine cytology is more important as a screening test, high sensitivity is desirable. If we used either two of the three key criteria, the diagnosis would have a much higher sensitivity of $79 \%$ and a decreased specificity of $91 \%$. To overcome this obstacle, other important features such as eccentric nucleus, nuclear molding and anisonucleosis were introduced. If we used two of the three key features plus either one of these other important features as the diagnostic standard, the diagnosis would have a slightly decreased sensitivity of $77 \%$ and a markedly increased specificity of $97 \%$. 
Table III. Comparison of Cytologic Features Present in Low-Grade Urothelial Carcinoma and Benign Lesions

\begin{tabular}{lcccc}
\hline & Specimen & $\begin{array}{c}\text { LGUC } \\
(\%)\end{array}$ & $\begin{array}{c}\text { Benign } \\
(\%)\end{array}$ & $P$ \\
\hline N/C ratio & Wash & 86.0 & 29.6 & $<0.01$ \\
& Urine & 55.3 & 10.7 & $<0.01$ \\
Cytoplasmic homogeneity & wash & 86.0 & 23.5 & $<0.01$ \\
& Urine & 51.3 & 10.7 & $<0.01$ \\
Irregular nuclear border & Wash & 78.0 & 11.8 & $<0.01$ \\
& Urine & 43.4 & 7.1 & $<0.01$ \\
Nuclear eccentricity & Wash & 82.0 & 17.6 & $<0.01$ \\
& Urine & 48.7 & 17.9 & $<0.01$ \\
Nuclear molding & Wash & 62.0 & 11.8 & $<0.01$ \\
& Urine & 27.6 & 3.6 & $<0.01$ \\
Anisonucleosis & Wash & 58.0 & 5.9 & $<0.01$ \\
& Urine & 18.4 & 0.0 & $<0.01$ \\
Hyperchromasia & Wash & 40.0 & 0.0 & $<0.01$ \\
& Urine & 27.6 & 3.6 & $<0.01$ \\
Elongated nucleus & Wash & 46.0 & 4.2 & $<0.01$ \\
& Urine & 17.1 & 0.0 & $<0.01$ \\
Absence of cytoplasmic collar & Wash & 36.0 & 5.9 & $<0.01$ \\
& Urine & 15.8 & 0.0 & $<0.01$ \\
Granular chromatin & Wash & 72.0 & 53.0 & $<0.01$ \\
& Urine & 39.5 & 14.3 & $<0.01$ \\
Cell cluster & Wash & 86.0 & 53.0 & $<0.01$ \\
& Urine & 42.1 & 32.1 & $<0.01$ \\
Necrosis & Wash & 8.0 & 0.0 & $<0.01$ \\
& Urine & 9.2 & 0.0 & $<0.01$ \\
\hline & & & &
\end{tabular}

LGUC, low-grade urothelial carcinoma.

Table IV. Sensitivity, Specificity, Positive Predictive Value, and Negative Predictive Value of Washing and Voided Urine Specimens

\begin{tabular}{lcccc}
\hline & $\begin{array}{c}\text { Sensitivity } \\
(\%)\end{array}$ & $\begin{array}{c}\text { Specificity } \\
(\%)\end{array}$ & $\begin{array}{r}P P V \\
(\%)\end{array}$ & $\begin{array}{r}N P V \\
(\%)\end{array}$ \\
\hline N/C ratio & 67.5 & 82.2 & 91.4 & 47.4 \\
Cytoplasmic homogeneity & 65.1 & 84.4 & 92.1 & 46.3 \\
Irregular nuclear border & 57.1 & 91.1 & 94.7 & 43.2 \\
Nuclear eccentricity & 61.9 & 82.2 & 90.7 & 43.5 \\
Nuclear molding & 41.3 & 93.3 & 94.5 & 36.2 \\
Anisonucleosis & 34.1 & 97.8 & 97.9 & 34.6 \\
Hyperchromasia & 32.5 & 97.8 & 97.6 & 34.1 \\
Elongated nucleus & 28.6 & 97.8 & 97.3 & 32.8 \\
Absence of cytoplasmic collar & 23.8 & 97.8 & 96.8 & 31.4 \\
Granular chromatin & 52.4 & 71.1 & 83.5 & 34.8 \\
Cell cluster & 59.5 & 60.0 & 80.6 & 34.6 \\
Necrosis & 8.7 & 100.0 & 100.0 & 28.1 \\
\hline
\end{tabular}

PPV, positive predictive value; NPV, negative predictive value; N/C, nuclear-to-cytoplasmic (ratio).

Although the three key cytologic features (increased N/C ratio, irregular nuclear membrane, and cytoplasmic homogeneity) had similar occurrences in ThinPrep samples, some cytologic alterations were noted in ThinPrep samples. In carcinoma cases, nuclear molding and anisonucleosis had much relative higher incidences in ThinPrep washing samples $(62 \%$ and $58 \%$ vs $6 \%$ and $27 \%$, respectively, in cytospin samples). In noncarcinoma cases, only $6 \%$ had open or vesicular nuclear chromatin compared with $22.0 \%$ in ThinPrep samples..$^{5}$ Nevertheless, this study confirms that despite such minor alterations ThinPrep produced well-pre-
Table V. Comparison of Cytologic Features Present in Low-Grade Urothelial Carcinoma and Benign Lesions

\begin{tabular}{lcccc}
\hline & Specimen & $\begin{array}{c}\text { LGUC } \\
(\%)\end{array}$ & $\begin{array}{c}\text { Benign } \\
(\%)\end{array}$ & $P$ \\
\hline High cellularity & Wash & 70.0 & 47.0 & 0.08 \\
& Urine & 46.1 & 42.9 & 0.20 \\
Open chromatin & Wash & 22.0 & 17.6 & 0.20 \\
& Urine & 10.5 & 3.6 & 0.50 \\
Acute inflammation & Wash & 22.0 & 47.0 & 0.35 \\
& Urine & 46.1 & 46.4 & 0.30 \\
Fragments irregular border & Wash & 2.0 & 0.0 & 0.05 \\
Prominent nucleoli & Urine & 3.9 & 0.0 & 0.55 \\
& Wash & 58.0 & 47.0 & 0.23 \\
Peripheral palisading & Urine & 15.8 & 25.0 & 0.75 \\
& Wash & 18.0 & 5.9 & 0.39 \\
& Urine & 5.3 & 0.0 & 0.29 \\
\hline
\end{tabular}

LGUC, low-grade urothelial carcinoma.

served samples with even distribution of cells and noticeable absence of most obscuring elements, such as blood, inflammation, and proteinaceous background. The diagnostic criteria derived from conventionally prepared samples in diagnosing LGUC would still be applicable in ThinPrep samples. In addition, as we showed in the study, although the incidences of diagnostic cytologic features were relatively low in the voided urine samples when compared with washing samples, they shared similar patterns. Therefore, the diagnostic criteria applicable to washings would also apply to voided urine samples.

\section{References}

1. Cancer Facts and Figures 2002. Atlanta, GA: American Cancer Society; 2002. p 4.

2. Reuter VE, Melamed MR. The urothelial tract: renal pelvis, ureter, urinary bladder, and urethra. In: Sternberg SS, Antonioli DA, Carter D, Mills SE, Oberman HA, editors. Diagnostic surgical pathology. 3rd ed. Philadelphia: Lippincott/Williams \& Wilkins; 1999. p 1869-1873.

3. Murphy WM, Beckwith JB, Farrow GM. Atlas of tumor pathology. Tumors of the kidney, bladder, and related urinary structures. 3rd series, fascicle 11. Washington, DC: Armed Forces Institute of Patholology; 1994. p 193-199.

4. Epstein J, Amin M, Reuter V, Mostofi FK. World Health Organization/ International Society of Urological Pathology consensus classification of urothelial (transitional) neoplasms of urinary bladder. Bladder Consensus Conference Committee. Am J Surg Pathol 2000;24:160-162.

5. Raab SS, Lenel JC, Cohen MB. Low grade transitional cell carcinoma of the bladder. Cancer 1994;74:1621-1626.

6. Raab SS, Slagel DD, Jensen CS, et al. Transitional cell carcinoma: cytologic criteria to improve diagnostic accuracy. Mod Pathol 1996; 9:225-232.

7. Awen C, Hathways S, Eddy W, Voskuil R, Janes C. Efficacy of ThinPrep preparation of cervical smears: a 1,000 case, investigatorsponsored study. Diagn Cytopathol 1994;11:33-36.

8. Fischler DF, Toddy SM. Nongynecologic cytology utilizing the ThinPrep processor. Acta Cytol 1996:40;669-675.

9. Michael CW, Hunter B. Interpretation of fine-needle aspirates processed by the ThinPrep technique: cytologic artifacts and diagnostic pitfalls. Diagn Cytopathol 2000;23:6-13. 
10. Leung CS, Chiu B, Bell V. Comparison of ThinPrep and conventional preparations: nongynecologic cytology evaluation. Diagn Cytopathol 1997; 16:368-371

11. Campbell MJ, Machin D. Medical statistics. West Sussex England: John Wiley \& Sons; 1990. p 63-78.

12. Le CT, Boen JR. Health and numbers: basic biostatistical methods. New York: Wiley-Liss; 1994. p 199-205.

13. DeMay RM. The arts and science of cytopathology: exfoliative cytology. Chicago: ASCP Press; 1996. p 385-410.

14. Badalament RA, Gay H, Gibas ES, et al. Monitoring endoscopic treatment of superficial bladder carcinoma by postoperative urinary cytology. J Urol 1987;138:760-762.
15. Murphy WM. Urinary cytology in diagnostic pathology. Diagn Cytopathol 1985;1:173-175.

16. Kannan V, Bose S. Low grade transitional cell carcinoma and instrument artifact: a challenge in urinary cytology. Acta Cytol 1993;37: 899-902.

17. Badalament RA, Hermansen DK, Kimmel M, et al. The sensitivity of bladder wash flow cytometry, bladder wash cytology, and voided cytology in the detection of bladder carcinoma. Cancer 1987;60:14231427.

18. Luthra UK, Pranab D, George J, Abdulla, MA, et al. Comparison of ThinPrep and conventional preparations: urine cytology evaluation. Diagn Cytopathol 1997;21:364-366. 